$$
1 N-39
$$

NASA Technical Memorandum 104052

8203

$p / 2$

\title{
Closed-Form Solutions For Linear Regulator Design Of Mechanical Systems Including Optimal Weighting Matrix Selection
}

Brantley R. Hanks and Robert E. Skelton

March 1991

\section{N/S \\ National Aeronautics and \\ Space Administration \\ Langley Research Center \\ Hampton, Virginia 23665}

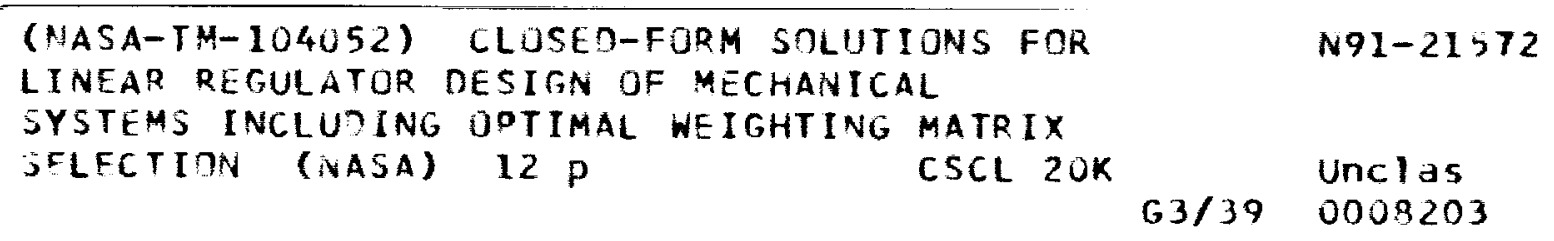




\section{CLOSED-FORM SOLUTIONS FOR LINEAR REGULATOR DESIGN OF MECHANICAL SYSTEMS INCLUDING OPTIMAL WEIGHTING MATRIX SELECTION}

\author{
Brantley R. Hanks* \\ NASA Langley Research Center \\ Hampton, Virginia
}

\author{
Robert E. Skelton** \\ Purdue University \\ West Lafayette, Indiana
}

\begin{abstract}
Vibrations in modem structural and mechanical systems can be reduced in amplitude by increasing stiffness, redistributing stiffness and mass, and/or adding damping if design tochniques are available to do so. Linear Quadratic Regulator (or LQR) theory in modern multivariable control design, attacks the general dissipative elastic system design problem in a global formulation. The optimal design, however, allows electronic connections and phase relations which are not physically practical or possible in passive structural-mechanical devices. This paper addresses the restriction of $L Q R$ solutions (to the Algebraic Riccati Equation) to design spaces which can be implemented as passive structural members and/or dampers. A general closed-form solution to the optimal free-decay control problem is presented which is tailored for structural-mechanical systems. The solution includes, as subsets, special cases such as the Rayleigh Dissipation Function and total energy. Weighting matrix selection is a constrained choice among several parameters to obtain desired physical relationships. The closed-form solution is also applicable to active control design for systems where perfect, collocated actuator-sensor pairs exist . Some examples of simple spring mass systems are shown to illustrate key points.
\end{abstract}

\section{Introduction}

Many modern structural and mechanical systems are subject to dynamic input forces which excite flexible vibrations. These vibations can be reduced in amplitude by increasing stiffness. However, reducing mass or inertia at the risk of reducing stiffness is an important factor for other reasons, such as launch costs or rigid-body control

\footnotetext{
* Head, Spacecraft Dynamics Branch Associate Fellow, AIAA

** Profesșor, Aeronautics and Astronautics Engincering, Fellow, AIAA
}

energy. A third design parameter, damping, offers an alternative for the stiffness-versus-mass dilemma, if suitable design methods can be developed for dissipative structures. Also, redistribution of stiffness and mass without increasing total mass can be beneficial if design techniques are available to do so.

Linear Quadratic Regulator (or LQR) theory in modern multivariable control design, attacks the general dissipative system design problem in a global formulation based first-order equations. This formulation usually yields a requirement for computer solution of a nonlinear matrix equation (the Algebraic Riccati Equation). For the case of structural and mechanical systems where secondorder governing equations predominate, the control equations are twice the usual matrix dimension, dependent on arbitrarily selected weighting matrices, and are difficult to interpret in terms of physical design parameters. In control design, a computer can implement connections and phase relations which are not physically practical or possible in the structural-mechanical case (e.g., Hanks and Skelton ${ }^{1}$, and Hvorat ${ }^{2}$ ). Nevertheless, the theory is applicable to passive structural systems and the challenge in using it is to restrict it to physically possible design spaces. Previous studies, such as Skelton, et. al. 3,4.5, have considered physically constrained computer solutions. Others, such as Kojitani and Ikeda ${ }^{6}$ and Belvin and Park ${ }^{7}$ have provided closed-form solutions for special cases.

This paper presents a general closed-form solution to the optimal free-decay control problem which is constrained to predetermined structural-mechanical geometry. It is derived entirely in physical coordinates using mass, stiffness, and viscous damping matrices. The solution results in a final (closed-loop) objective function which is a generalized energy expression for dissipative structural-mechanical systems. Application is discussed to special cases including the Rayleigh Dissipation Function, total energy, and known closed-form solutions for particular objective functions. Weighting matrix selection is a choice among several parameter matrices. For design objectives commonly used in $\mathrm{LQR}$ control system design (i.e., choices of $Q$ weighting matrices), direct solution for optimal design parameters ( $R$ weighting matrices) is possible, but frequently structurally impractical. The closed-form solution and its physical interpretation is also applicable to the active control problem for similar systems where perfect, collocated actuator-sensor pairs cxist and, hence, is applicable for initial control design and 


\section{IL. Connectivity Modeling of Discrete Physical Systems}

Any discrete physical system, or discrete representation of a continuous physical system, may be modeled in connectivity form, that is, as a three-matrix product $\mathcal{B G H}$ where each column of $\mathcal{B}$ and each row of $\mathrm{H}$ carries connection geometry information and $G$ is a diagonal "gain" matrix. G has dimension mxm where $m$ is the number of connections between nodes, $B$ has dimension $\mathbf{n x m}$ where $\mathbf{n}$ is the number of input (forced) nodes and $\mathrm{H}$ has dimension $\mathrm{mxk}$ where $\mathrm{k}$ is the number of output (sensed) nodes. In passive structural/mechanical systems, or active and mixed active-passive systems with perfect collocated sensors and actuators, $\mathrm{H}=\mathcal{B}^{*}$, where * denotes the complex conjugate transpose of $B$. Thus, the usual nxn symmetric mass, stiffness, and damping parameter matrices, $\mathcal{M}, \mathcal{D}$, and $\mathcal{X}$ become

$\mathcal{M}=\mathcal{B}_{m} G_{-} \mathcal{B}_{-}^{*}, \quad \mathcal{D}=\mathcal{B}_{i} \mathrm{G}_{i} \mathcal{B}_{i}^{*}$, and $\mathcal{X}=\mathcal{B}_{k} \mathrm{G}_{k} \mathcal{B}_{k}^{*}$

where

$$
\mathrm{G}_{\mathrm{j}}=\operatorname{diag}\left[\cdots \mathrm{g}_{1} \cdots\right]_{1}
$$

are $m x m$ diagonal matrices. The number of terms, $g_{i}$, on the diagonal of $\mathrm{G}$ in each parameter is large, i.e., on the order of $n+(p / 2)$, where $p$ is the number of non-zero offdiagonal terms in each matrix. It is convenient to $\operatorname{kecp} \mathcal{B}$ the same for all three matrices and enter $\mathrm{g}_{j}=0$ for nonexistent connections, provided there is no requirement for

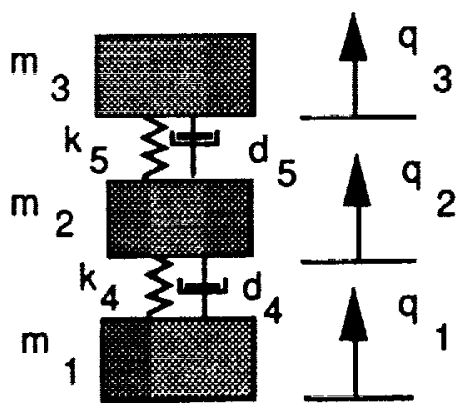

Fig. 1 Simple Floating Three-Mass Elastic System

the inverse of $G$ to exist. The dimension of $G$ is usually determined by the number of stiffness connections in $\mathcal{K}$. The columns, $b_{i}$ of $B$ contain one or two non-zero entries of dircction cosines to ground or betwcen nodes. The diagonal tcrms of each $\mathrm{G}$ matrix contains local parameter magnitudes between nodes such as lumped mass, spring or damper coefficients in spring-mass systems or, similarly, truss elongation stiffness in trusses. For illustration, consider the simple system of Fig. 1. For this system, the parameter matrices are as follows:

$$
\mathcal{B}_{-}=\mathcal{B}_{1}=\mathcal{B}_{4}=\left[\begin{array}{ccccc}
1 & 0 & 0 & 1 & 0 \\
0 & 1 & 0 & -1 & -1 \\
0 & 0 & 1 & 0 & 1
\end{array}\right] \text {, }
$$

$$
\begin{aligned}
\mathrm{G}_{\mathcal{M}} & =\left[\begin{array}{ccccc}
m_{1} & 0 & 0 & 0 & 0 \\
0 & m_{2} & 0 & 0 & 0 \\
0 & 0 & m_{3} & 0 & 0 \\
0 & 0 & 0 & 0 & 0 \\
0 & 0 & 0 & 0 & 0
\end{array}\right] \\
\mathrm{G}_{x} & =\left[\begin{array}{lllll}
0 & 0 & 0 & 0 & 0 \\
0 & 0 & 0 & 0 & 0 \\
0 & 0 & 0 & 0 & 0 \\
0 & 0 & 0 & k_{1} & 0 \\
0 & 0 & 0 & 0 & k_{2}
\end{array}\right],
\end{aligned}
$$

and $G_{v}$ has the same form as $G_{x}$. If compactness is important, or if inverses of $G$ are necessary, these matrices may be simplified by eliminating the zero-gain columns and rows and the corresponding columns of $B$, but the form here is useful for explanation of relative positions of terms. Furthermore, if the springs and dampers are active devices, or are in parallel with active devices, the net gain terms can be negative or zero.

In this paper, we will consider two types of gains the initial system gains (i. e. $\mathcal{M}, \mathcal{X}$, and $\mathcal{D}$ L and "passive control" gains ( changes in mass, $\Delta \mathcal{M}$, stiffness, $\Delta X$, or damping, $\Delta \mathcal{D}$ ). A control gain may involve changing an existing gain value or introducing a new connection which was not present in the initial design. The term closed-loop gains, denoted by a hatted symbol, will refer to the final mass, stiffness, and damping (i.c., $\hat{\mathcal{M}}=\mathcal{M}+\Delta \mathcal{M}, \quad \hat{\mathcal{X}}=\mathcal{X}+\Delta \mathcal{X}$, and $\hat{\mathcal{D}}=\mathcal{D}+\Delta \mathcal{D}$ ), after either passive or active changes are made. Active controls using acceleration, position, and rate feedback effectively produce changes in mass, stiffness, and damping, respectively (assuming negligible actuator and sensor dynamics).

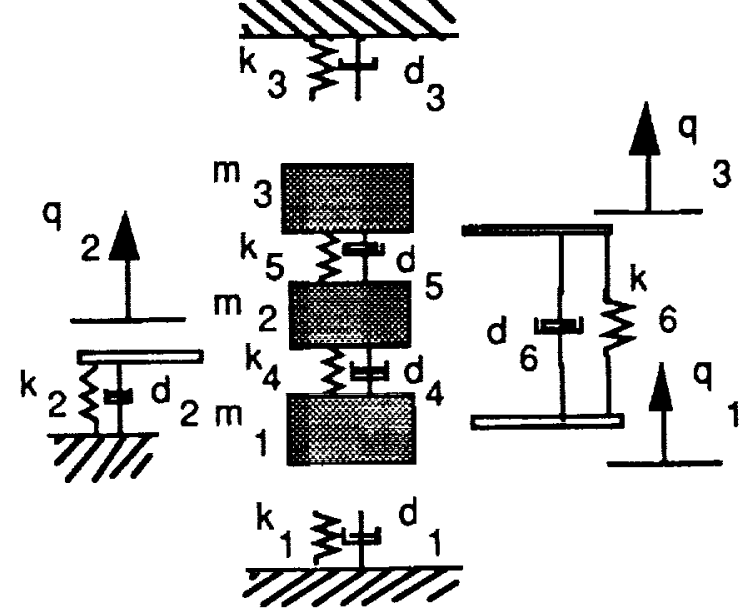

Fig. 2 Three-Mass Floating Elastic System with Possible Collocated Control Connections 
In designing a set of springs (passive positionfeedback) and dampers (passive rate-feedback), a set of active position, rate and acceleration feedback controls, or a combination of all, a large number of connections are possible. For the example of Fig. 1, the four possible additional connection paths for collocated pairs are shown schematically in Fig. 2. There are 24 possible connection combinations for the system of Fig. 2, two of which are shown with their corresponding $\mathcal{B}$ matrices in Fig. 3 . Configuration 1 includes all possible (collocated) connections and represents the type of solution which may be obtained from standard, unconstrained LQR controls designs. In such designs, interconnection capability is assumed available at all nodes but, especially in the case of passive implementation, is rarely practical.
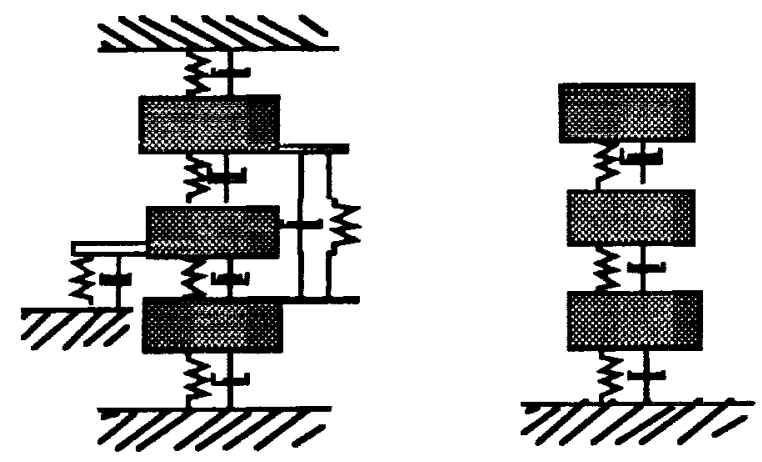

$$
\mathcal{B}=\left[\begin{array}{cccccc}
1 & 0 & 0 & 1 & 0 & -1 \\
0 & 1 & 0 & -1 & -1 & 0 \\
0 & 0 & 1 & 0 & 1 & 1
\end{array}\right]
$$

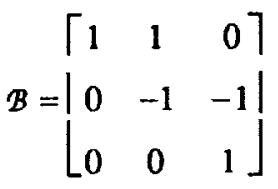

Configuration 2
Fig.3 Some Altemative Connection Geometries for a 3-Mass Simple System

Hanks and Skelton ${ }^{1}$, using a finite-element, cantilever beam example, showed that the addition of connections from analysis nodes to ground and between non-adjacent analysis nodes are required by an LQR design solution. Such connection freedom may not be costeffective, even if it is possible. Hvorat ${ }^{2}$, studying minimization of squared displacements on a two-mass, simple system, concluded from mathematical critcria that an LQR controller could not be implemented by a single, passive, non-grounded controller. The option to constrain the solution to allow only physically practical connection locations, such as in Configurations 1 and 2 in Fig. 3, is necessary in practical passive or active control design. Skelton, et. al. 3,4 , using the above diagonal-gain connectivity approach to fix control configuration geometry, applied an iteralive constrained output feedback solution to minimize a quadratic objective function while satisfying predetermined limits on parameter changes. The following sections discuss a closed-form solution to the LQR problem which provides the optimal solution while maintaining predefined passive, or collocated active, control geometry. It is not restricted to the diagonal gain formulation, but certain parameters such as weighting matrix values, can be physically interpreted using the connectivity concept.

\section{Theory Preliminaries}

Linear Quadratic Regulator (LQR) control theory has potential for structural dynamics design in mechanics. This theory is widely available in textbooks, such as Skelton ${ }^{5}$, but an outline is presented here for purposes of reference and terminology in deriving later results. Freedecay and full-state feed-back motion will be discussed.

The time-invariant LQR approach minimizes an objective function, $V$, which is the infinite-time integral of the sum of weighted quadratic potential function of the state vector, $z$, and the control vector, $u$. It is well known that the minimization of

$$
\mathrm{V}=\frac{1}{2} \int_{0}^{-}(\mathrm{z} * \mathrm{Qz}+\mathrm{u} * \mathrm{Ru}) \mathrm{dt}
$$

subject to the constraints of the system equations of motio

$$
\dot{\mathrm{z}}=\mathrm{Az}+\mathrm{Bu}
$$

yiclds

$$
\mathrm{u}_{\mathbf{q}}=-\mathrm{R}^{-1} \mathrm{~B} * \mathrm{Kz}
$$

where $\mathrm{K}$ is a symmetric, positive-definite gain matrix which satisfies

$$
K A+A * K-K B R^{-1} B K+Q=0
$$

Substituting the linear optimal feedback control (5) into (4), the closed-loop system equations of motion become

$$
\dot{z}=A z-B R^{-1} B K z=(A+\Delta A) z=\hat{A} z
$$

Similarly, (3) yields the optimal objective function

$$
\begin{aligned}
\mathrm{V} & =\frac{1}{2} \int_{0}^{-}\left(\mathrm{z}^{*} \mathrm{Qz}+\mathrm{z}^{*} \mathrm{KBR}^{-1} \mathrm{BKz}\right) \mathrm{dt} \\
& =\frac{1}{2} \int_{0}^{-} \mathrm{z} *(\mathrm{Q}+\Delta \mathrm{Q}) \mathrm{zdt} \\
& =\frac{1}{2} \int_{0}^{-}\left(\mathrm{z}^{*} \hat{\mathrm{Q}} \mathrm{z}\right) \mathrm{dt}=\frac{1}{2} \mathrm{z}^{*}(0) \mathrm{Kz}(0) \\
\hat{\mathrm{Q}} & =\mathrm{Q}+\mathrm{KBR}^{-1} \mathrm{~B} * \mathrm{~K}
\end{aligned}
$$

where $\mathrm{z}(0)$ is the initial state vector.

If $(A, B)$ is stabilizable (that is, the uncontrollable modes are stable) and $(A, Q)$ is detectable (that is, the unobservable modes are stable), then the feedback gain matrix, $K$, that solves (6) is positive semi-definite and $\hat{A}=A+\Delta A$ is also stable, Equation (6) can also be written 


$$
K \hat{A}+\hat{A}^{*} K+\hat{Q}=0
$$

From Liapunov stability theory, equation (9) represents a stable system if $K$ is positive definite and $\hat{Q}$ is positive semi-definite, that is, $K>0, \hat{Q} \geq 0$.

Now, consider the familiar equation

$$
\mathscr{M} \ddot{q}+(\mathcal{D}+\mathcal{G}) \dot{q}+\mathcal{X q}=\mathcal{B u}
$$

where $\mathcal{M}, \mathcal{X}, \mathcal{D}$, and $\mathcal{G}$ are mass, stiffness, damping and gyroscopic-coupling matrices, respectively, having the following properties:

$$
\begin{aligned}
& \mathcal{M}=\mathcal{M}^{*}>0, \quad \mathcal{X}=\mathcal{X}^{*} \geq 0, \\
& \mathcal{D}=\mathcal{D}^{*} \geq 0, \text { and } G=-G^{*}
\end{aligned}
$$

By writing the state $z$, in (4) as

$$
z=\left\{\begin{array}{c}
q \\
\dot{q}
\end{array}\right\}
$$

where $q$ is displacement and $\dot{q}$ is velocity, the equations of motion (10) become, in the first-order form of (4),

$$
\begin{aligned}
\dot{z} & =\left\{\begin{array}{c}
\dot{q} \\
\ddot{q}
\end{array}\right\}=(A+\Delta A) z=\hat{A} z \\
& =\left[\begin{array}{cc}
0 & I \\
-\mathscr{M}^{-1} \mathcal{X} & -\mathscr{M}^{-1}(\mathcal{D}+G)
\end{array}\right]\left\{\begin{array}{l}
q \\
\dot{q}
\end{array}\right\}+\left[\begin{array}{c}
0 \\
\mathcal{M}^{-1} \mathcal{B}
\end{array}\right] u
\end{aligned}
$$

The optimal control (5) is

$$
\begin{aligned}
u_{q u} & =-R^{-1}\left[\begin{array}{ll}
0 & \mathcal{B}^{*} \mathcal{M}^{-1}
\end{array}\right]\left[\begin{array}{ll}
\mathrm{K}_{11} & \mathrm{~K}_{12} \\
\mathrm{~K}_{21} & \mathrm{~K}_{22}
\end{array}\right]\left\{\begin{array}{l}
\mathrm{q} \\
\dot{q}
\end{array}\right\} \\
& =-\mathrm{R}^{-1} \mathcal{B}^{*} \mathcal{M}^{-1}\left(\mathrm{~K}_{21} \mathrm{q}+\mathrm{K}_{2} \dot{\mathrm{q}}\right)
\end{aligned}
$$

and the objective function (3) has the form

$$
V=\frac{1}{2} \int_{0}^{-}\left\{(q u) *\left[\begin{array}{ll}
Q_{11} & Q_{12} \\
Q_{21} & Q_{22}
\end{array}\right]\left\{\begin{array}{c}
q \\
\dot{q}
\end{array}\right\}+u * R u\right\} d t
$$

Substituting the optimal control (14) into (10) gives the equations of motion of the closed-loop, or revised system

$$
\begin{aligned}
\mathscr{M} \overline{\mathrm{q}}+\left(\mathcal{D}+\mathcal{G}+\mathscr{B R}{ }^{-1} \mathcal{B}^{*} \cdot \mathcal{M}^{-1} \mathrm{~K}_{2}\right) \dot{\mathrm{q}} \\
+\left(\mathcal{X}+\mathcal{B} \mathrm{R}^{\cdot} \mathcal{B}^{*} \mathcal{M}^{-1} \mathrm{~K}_{21}\right) \mathrm{q}=0
\end{aligned}
$$

Similarly, the closed-loop integrand $z * \hat{Q} z$ of the optimal objective function (15) becomes

$$
z^{*} \dot{\mathrm{Q} z}=\mathrm{z}^{*} \mathrm{Qz}+\mathrm{u} * \mathrm{Ru}=\mathrm{z} *\left(\mathrm{Q}+\mathrm{KBR} \mathrm{R}^{4} \mathrm{BK}\right) \mathrm{z}
$$

where the $2 n \times 2 n$ matrix Qreduces to

$$
\hat{\mathrm{Q}}=\left[\begin{array}{ll}
\mathrm{Q}_{11} & \mathrm{Q}_{2} \\
\mathrm{Q}_{\mathrm{n}} & \mathrm{Q}_{\mathrm{z}}
\end{array}\right]+\left[\begin{array}{l}
\mathrm{K}_{12} \mathcal{M}^{-1} \mathcal{B}^{\cdot} \\
\mathrm{K}_{2} \mathcal{M}^{-1} \mathcal{B}^{\cdot}
\end{array}\right] \mathrm{R}^{-1}\left(\mathcal{B M}^{-1} \mathrm{~K}_{21} \quad \mathcal{M M}^{-1} \mathrm{~K}_{\mathrm{z}}\right)
$$

Now, for simplification of notation, we define

$$
\Gamma=\mathbb{R}^{-1} \mathcal{B}^{*}
$$

The $2 n \times 2 n$ Riccati equation, (6), from which the control gains are derived, can be written in second-order form as four $n \times n$ matrix equations of which three are independent ${ }^{5}$. These three, including the notation (19), are as follows:

$$
\begin{gathered}
-K_{12} \mathcal{M}^{-1} \mathcal{X}-\mathcal{X M}^{-1} \mathrm{~K}_{21}-\mathrm{K}_{12} \mathcal{M}^{-1} \Gamma \mathcal{M}^{-1} \mathrm{~K}_{21}+\mathrm{Q}_{11}=0 \\
-\mathrm{K}_{22} \mathcal{M}^{-1}(\mathcal{D}+\mathcal{G})-(\mathcal{D}+G) * \mathcal{M}^{-1} \mathrm{~K}_{22} \\
\quad+\mathrm{K}_{12}+\mathrm{K}_{21}-\mathrm{K}_{22} \mathcal{M}^{-1} \Gamma \mathcal{M}^{-1} \mathrm{~K}_{22}+\mathrm{Q}_{22}=0
\end{gathered}
$$

$\mathrm{K}_{11}-\mathrm{K}_{12} \mathcal{M}^{-1}(\mathcal{D}+G)-\mathcal{X M}^{-1} \mathrm{~K}_{22}-\mathrm{K}_{12} \mathcal{M}^{-1} \Gamma \mathcal{M}^{-1} \mathrm{~K}_{22}+\mathrm{Q}_{12}=0$

\section{IY. Closed-Form Solutions of Algebraic Biccati Equations}

Equation (16) provides the basic relationships between the physical system parameters and the mathematical design parameter matrices. These are as follows:

$$
\Delta \mathcal{X}=\Gamma \mathcal{M}^{-1} \mathrm{~K}_{21}, \quad \Delta(\mathcal{D}+\mathcal{G})=\Gamma \mathcal{M}^{-1} \mathrm{~K}_{22}
$$

Hence, solution of (20) through (22) to obtain $\mathrm{K}_{12}$ and $\mathrm{K}_{22}$ provides the optimal system stiffness and damping changes for any permissible set of $Q$ and $R$ matrices. Usually, these equations are solved numerically by computer. However, closed-form solutions are known for some special cases as discussed in the following section.

\section{The Solution of Koiitani and_Ikeda}

Kojitani and Ikeda ${ }^{6}$, propose the following wcighting matrix:

$$
\mathrm{Q}=\mathrm{a}\left[\begin{array}{cc}
2 \mathcal{X}+\mathrm{a} \Gamma & \mathcal{G} \\
\mathcal{G}^{*} & \frac{\mathrm{b}}{\mathrm{a}}(2 \mathcal{D}+\mathrm{b} \Gamma)-2 \mathcal{M}
\end{array}\right]
$$

Where $a$ and $b$ are arbitrary scalars which must be restricted for existence of the Riccati equation solution. This results in an exact solution to the Riccati equation of the form 


$$
\mathbf{K}=\mathrm{b}\left[\begin{array}{cc}
\mathcal{X}+\mathrm{a} \Gamma+\frac{\mathrm{a}}{\mathrm{b}} \boldsymbol{D} & \frac{\mathrm{a}}{\mathrm{b}} \mathcal{M} \\
\frac{\mathrm{a}}{\mathrm{b}} & \mathcal{M}
\end{array}\right], \quad \mathrm{b}>0
$$

where the submatrices

$$
\mathrm{K}_{12}=\mathrm{K}_{21}=\mathrm{aM}, \quad \mathrm{K}_{22}=\mathrm{b} \mathcal{M}
$$

lead to the very simple control vector

$$
u=-R^{-1} \mathcal{B}^{*}(\mathrm{aq}+\mathrm{b} \dot{q})=-R^{-1} \mathcal{B}^{*}[\text { al } \vdots \text { bl }]\left\{\begin{array}{l}
q \\
\dot{q}
\end{array}\right\}_{(27)}
$$

Proof that (25) is a solution is a simple matter of substituting the terms into (20) through (22).

Assuming that $\Gamma$ is preassigned, the above solution to (20) through (22) has two arbitrary parameters $a$ and $b$, resulting in one added constraint, $K_{21}=(a / b) K_{22}$. This constraint requires that the stiffness change be proportional to the damping change. Replacing this constraint by the condition $a=0$ is also possible. ( $b=0$ is not permissible if $K$ is to be positive definite.) It also provides a direct implementation of control feedback loops in which measurements are preweighted by a scalar

constant rather that the matrices $\mathcal{M}^{-1}$ and/or $\mathrm{K}_{\mathrm{ij}}$. This allows observation of a very important point. The closedloop system equations of motion are

$$
\begin{aligned}
\ddot{M} \bar{q}+(\mathcal{D} & +\mathcal{G}+\mathrm{b} \Gamma) \dot{\mathrm{q}}+(\mathcal{X}+\mathrm{a} \Gamma) \mathrm{q} \\
& =\ddot{\mathcal{M}} \ddot{\mathrm{q}}+(\mathcal{D}+\mathcal{G}+\Delta \mathcal{D}) \dot{\mathrm{q}}+(\mathcal{X}+\Delta \mathcal{X}) \mathrm{q}=0
\end{aligned}
$$

From (28), it is clear that the closed-loop stiffness and damping matrix changes are entirely a function of free parameters $(a, b, \Gamma$.). The optimal control design has bcen obtained and its form is predetermined by the scalar constants $\mathrm{a}$ and $\mathrm{b}$ and the matrix $\Gamma$ (composed of the assumed $\mathcal{B}$ and $\mathbf{R}$ ). Even with the luxury of full-state feedback, we have no indication of how to choose the key parameters which define the optimal controller design (i.e., stiffness and damping). In fact, the following proposition can be stated by inspection of (26) through (28):

Proposition: Any solution of the full-state feedback control problem which results in Riccati solutions of the form (26) transforms the problem into the symmetric form of the standard measurement feedback problem, with $R^{-1}$ being the equivalent of the optimal gain sought.

Problem: Applying the diagonal gain formulation of Section $I, R^{-I}$ becomes a diagonal gain matrix whose diagonal elements $\mathrm{r}_{\mathrm{i}}^{-1}$ are the gains of single physical connections. For any sel of mechanical/structural connections, find an optimal $R$ matrix which provides the gains for the optimal control problem.

Detcrmination of this $\mathbf{R}$ matrix, will be explored in Section V.

\section{The Solution of Belvin and Park.}

In the above solution, $Q$ contains terms of the optimal control which is being sought. A solution which does not have this characteristic was offered by Belvin and Park ${ }^{7}$ for the special case where $\mathcal{B}$ is square and of full rank. Their closed-form solution to (20) through (22) assumes $Q$ and $R$ as follows:

$$
\mathrm{Q}=\left[\begin{array}{cc}
\alpha \mathcal{X} & 0 \\
0 & \beta \mathcal{M}
\end{array}\right], \quad \mathrm{R}=\mathcal{B}^{*} \mathcal{K}^{-1}
$$

The solution is presented in the following form:

$$
\mathrm{K}=\left[\begin{array}{cc}
2 \zeta \Psi & \eta \mathcal{M} \\
\eta \mathcal{M} & \zeta \mathcal{M} \mathcal{K}^{-1} \Psi
\end{array}\right]
$$

Where $\alpha$ and $\beta$ are arbitrary scalar constants and

$$
\eta=\sqrt{1+\alpha}-1, \quad \varsigma=\sqrt{2 \eta+\beta},
$$

and $\Psi=\mathcal{M}^{\frac{1}{2}}\left(\mathcal{M}^{\frac{1}{2}} \mathcal{X} \mathcal{M}^{\frac{1}{2}}\right)^{\frac{1}{2}} \mathcal{M}^{\frac{1}{2}}=\mathcal{M}^{\frac{1}{2}} \mathcal{D}_{\alpha} \mathcal{M}^{\frac{1}{2}}$

$\left(\mathcal{D}_{\text {cr }}\right.$ denotes the critical damping matrix ${ }^{8}$.) This solution yiclds a control law

$$
B u=-\eta X q-\zeta \dot{q}
$$

which minimizes the sum of the strain and kinetic energies of the open-loop system. It is interesting to note that for a single-degree-of-freedom system the velocity feedback gain is $\varsigma \sqrt{k m}$, that is, proportional to critical damping.

\section{A General Energy-Related Solution}

Equations (20) to (22), the Riccati sub-equations, provide three relationships among the design parameter matrices. There are seven undefined parameter matrices in these equations, $3 \mathrm{Q}_{\mathrm{ij}}$ 's, $3 \mathrm{~K}_{\mathrm{ij}}$ 's and $\mathrm{r}$. Picking any four defines a unique relationship among the remaining matrices. It is commonly assumed, as in the KojitaniIkeda and Belvin-Park solutions, that $\Gamma$ and $Q_{i j}$ are preassigned and the equations solved for $K$. ( $\Gamma$ is actually composed of two matrices, $\mathcal{B}$ and $\mathrm{R}$, but they never appear independently in the above equations and may be considered as a unit in the solution, with the condition that $\mathrm{R}^{-1}$ must exist.) This is not required, however. Choosing physically meaningful and mathematically permissible forms for other combinations of four terms is possible. A great many logical combinations of parameters exist, some of which may take the form of constraint equations.

The physically simple control vector form (27) of the Kojitani-Ikeda solution suggests a meaningful choice 
for two of the parameters, $K_{12}$ and $K_{22}$. Therefore, substituting (27) into (20) through (22), we obtain a general energy-related solution set of which other solutions are special cases obtained by different choices of any permissible set of two of the remaining parameters. This solution is stated in theorem form as follows:

Theorem I The Algebraic Riccati Equation for second-order mechanical systems is uniquely solved in closed form by the open-loop matrix pair

$$
\begin{gathered}
\mathrm{Q}=\left[\begin{array}{cc}
2 \mathrm{aX}+\mathrm{a}^{2} \Gamma & \Pi+\mathrm{ab} \Gamma \\
\Pi^{*}+\mathrm{ab} \Gamma & 2 \mathrm{~b} \mathcal{D}+\mathrm{b}^{2} \Gamma-2 \mathrm{a} \hat{\mathcal{M}}
\end{array}\right] \\
\mathrm{K}=\left[\begin{array}{cc}
\hat{\Theta} & \mathrm{a} \hat{\mathcal{M}} \\
\mathrm{a} \hat{\mathcal{M}} & \mathrm{b} \dot{\mathcal{M}}
\end{array}\right]
\end{gathered}
$$

where $\Pi=-\hat{\Theta}+b \mathcal{X}+\mathrm{a}(\mathcal{D}+\mathcal{G})$. For $\mathrm{K}$ to be positive definite, the parameters of (33) are subject to the following constraints:

$\mathrm{K}_{2 \mathrm{z}}>0 \Rightarrow \mathrm{b}>0 ; \quad\left(\mathrm{K}_{11}-\mathrm{K}_{\mathrm{zz}} * \mathrm{~K}_{\mathrm{z}}^{-\mathrm{T}} \mathrm{K}_{\mathrm{1z}}\right)>0 \Rightarrow \hat{\theta}>\frac{\mathrm{a}^{\mathrm{d}}}{\mathrm{b}} \mathcal{M}$

Note that the constraints (34) require a damping increase but, in general, allow positive, negative or zero stiffness change.

The solution of Theorem 1 contains all possible scts of solutions to the optimal full-state-feedback control problem in which collocated perfect sensor-actuator pairs arc present. This includes all combinations of linear structures and linear viscous dampers. The precise member (actuator-sensor pair) locations are set by the connection matrix $\mathcal{B}$. The solution is best interpreted physically by regrouping terms in the closed-loop form of the original objective function as follows:

$$
\begin{aligned}
& \mathrm{V}=\frac{1}{2} \int_{0}^{-}(\mathrm{z} * \mathrm{Q} \mathrm{z}+\mathrm{u} * \mathrm{Ru}) \mathrm{dt}= \\
& \frac{1}{2} \int_{0}^{-}\left\{\begin{array}{r}
\left(\begin{array}{c}
q \\
q
\end{array}\right)^{*}\left[\begin{array}{cc}
2 a \mathcal{X}+a^{2} \Gamma & \Pi+a b \Gamma \\
\Pi^{*}+a b \Gamma & 2 b \mathcal{D}+b^{2} \Gamma-2 a \hat{\mathcal{M}}
\end{array}\right]\left\{\begin{array}{l}
q \\
\dot{q}
\end{array}\right\} \\
+\left(\begin{array}{ll}
q & \dot{q}
\end{array}\right)^{*}\left[\begin{array}{cc}
a^{2} \Gamma & a b \Gamma \\
a b \Gamma & b^{2} \Gamma
\end{array}\right]\left\{\begin{array}{l}
q \\
\dot{q}
\end{array}\right\}
\end{array}\right\}
\end{aligned}
$$

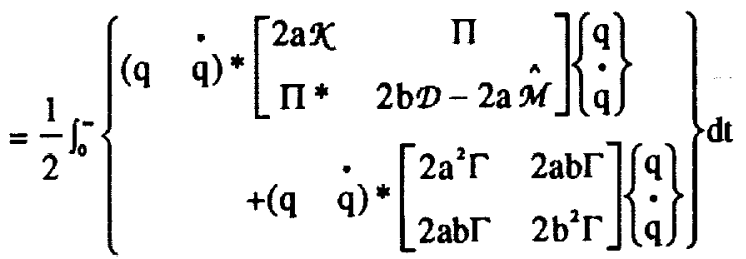

$$
\begin{aligned}
& =\frac{1}{2} \int_{0}^{-}\left(\begin{array}{ll}
\mathrm{q} & \dot{\mathrm{q}}
\end{array}\right)^{*}\left[\begin{array}{cc}
2 \mathrm{a} \hat{\mathcal{X}} & 2 \hat{\Pi} \\
2 \hat{\Pi} * & 2 \mathrm{~b} \hat{\mathcal{D}}-2 \mathrm{a} \hat{\mathcal{M}}
\end{array}\right]\left\{\begin{array}{l}
\mathrm{q} \\
\dot{\mathrm{q}}
\end{array}\right\} \mathrm{dt}
\end{aligned}
$$

$$
\text { where } \hat{\Pi}=-\hat{\Theta}+b \hat{\mathcal{X}}+a\left(\hat{\mathcal{D}}+\mathcal{G}^{*}\right)
$$

Using the closed-loop formulation of (35),

Theorem 1 can be alternatively stated as follows:

Theorem 2 The Algebraic Riccali Equation for second-order mechanical systems is uniquely solved in closed form by the closed-loop matrix pair

$$
\begin{gathered}
\hat{\mathrm{Q}}=\left[\begin{array}{cc}
2 \mathrm{a} \hat{\mathcal{X}} & -\hat{\Theta}+\mathrm{b} \hat{\mathcal{X}}+\mathrm{a}(\hat{\mathcal{D}}+G) \\
-\hat{\Theta}+\mathrm{b} \hat{\mathcal{X}}+\mathrm{a}\left(\hat{\mathcal{D}}+\mathcal{G}^{*}\right) & 2 \mathrm{~b} \dot{\mathcal{D}}-2 \mathrm{a} \hat{\mathcal{M}}
\end{array}\right] . \\
\mathrm{K}=\left[\begin{array}{cc}
\hat{\Theta} & \mathrm{a} \hat{\mathcal{M}} \\
\mathrm{a} \dot{\mathcal{M}} & \mathrm{b} \hat{\mathcal{M}}
\end{array}\right], \quad \hat{\mathrm{Q}} \geq 0, \mathrm{~K}>0
\end{gathered}
$$

where, $\hat{\theta},\left(\hat{\Theta}=K_{11}\right)$ is an arbitrary matrix and $a$ and $b$ are arbitrary scalar consiants. For $K$ to be positive definite, the parameters of (35) are subject to the constraints (34).

For stability, (9) requires $\hat{Q} \geq 0$ which translates, using the approach of (34), to

$$
\mathrm{a} \geq 0 \text { and } \hat{\mathcal{D}}>\frac{\mathrm{a}}{\mathrm{b}} \hat{\mathcal{M}}
$$

a very conservative requirement unless $\mathrm{a}^{2}$ is small relative to $b$. The above results are collectively summarized in the following theorem:

Theorem 3 All control laws which can be implemented by collocated actuator-sensor pairs, including structural stiffness or linear viscous dampers, to minimize

$$
\mathrm{V}=\frac{1}{2} \int_{0}^{-}\left(\mathrm{z}^{*} \mathrm{Qz}+\mathrm{u}^{*} \mathrm{Ru}\right) \mathrm{dt}, \quad \mathrm{Q} \geq 0, \quad \mathrm{R}>0
$$

subject to the equations of motion

$$
\mathscr{M} \ddot{q}+(\mathcal{D}+\mathcal{G}) \dot{q}+X q=\mathcal{B u}, \quad z=\left\{\begin{array}{c}
q \\
\dot{q}
\end{array}\right\}
$$

are given by

resulting in

$$
\mathbf{u}=-\mathrm{aR}^{\cdot 1} \mathcal{B}^{*} \mathrm{q}+\mathrm{bR}^{-1} \mathcal{B}^{*} \dot{\mathrm{q}}
$$

$$
B u=-a_{B} R^{-1} B^{*} q-b B^{-1} \mathcal{B}^{*} \dot{q}=\Delta X q+\Delta \mathcal{D} \dot{q}
$$

where $a$ and $b$ are scalar consiants.

The closed-loop objective function is 
$\mathrm{V}=\frac{1}{2} \int_{0}^{\sigma}\left(\begin{array}{ll}\mathrm{q} & \dot{\mathrm{q}}\end{array}\right)^{*}\left[\begin{array}{cc}2 \mathrm{a} \hat{\mathcal{X}} & \hat{\Pi} \\ \hat{\Pi} * & 2 \mathrm{~b} \hat{\mathcal{D}}-2 \mathrm{a} \hat{\mathcal{M}}\end{array}\right]\left\{\begin{array}{l}\mathrm{q} \\ \dot{\mathrm{q}}\end{array}\right\} \mathrm{dt}$

where $\hat{\Pi}=-\hat{\Theta}+b \hat{X}+a(\hat{\mathcal{D}}+\mathcal{G})$ and the value of $V$ is

$V=\frac{1}{2} z *(0) K z(0)$

$$
=\frac{1}{2}\left(q^{*}(0) \quad \dot{q}^{*}(0)\right)\left[\begin{array}{cc}
\hat{\Theta} & \mathrm{a} \hat{\mathscr{M}} \\
\hat{\mathrm{a}} & \mathrm{b} \hat{\mathcal{M}}
\end{array}\right]\left\{\begin{array}{l}
\mathrm{q}^{*}(0) \\
\dot{q}^{*}(0)
\end{array}\right\}
$$

where $\hat{\theta}$ is a symmetric, positive-definite matrix. The parameters $a, b$, and $\hat{\Theta}$ are arbitrary but subject to the following constraints for a valid (i.e., $K>0$ ) and stable

(i.e., $\hat{Q} \geq 0$ )solution:

$\mathrm{b}>0$ and $\hat{\Theta}>\frac{\mathrm{a}^{2}}{\mathrm{~b}} \hat{\mathcal{M}} ; \quad \mathrm{a} \geq 0$ and $\hat{\mathcal{D}}>\frac{\mathrm{a}}{\mathrm{b}} \hat{\mathcal{M}}$

The integrand of (38) is a "generalized-energy" function for linear, viscously-damped, multi-variable mechanical systems which are altered by changing member damping and stiffness. For $\hat{\Theta}=b \hat{\mathcal{X}}, a=1 / 2$, and $\hat{\mathcal{D}}=0$, (3) reduces to the standard Lagrangian for conservative systems. However, since $b>0$ is required for a solution to exist, the closed-loop damping cannot be zero and a direct extrapolation to the Lagrangian is not valid. The expression for the control forces or system changes,

$\left(\mathcal{B} \mathrm{u}=-\mathrm{a} B \mathrm{R}^{-1} \mathcal{B}^{*} \mathrm{q}-\mathrm{b} B \mathrm{R}^{-1} \mathcal{B}^{*} \dot{\mathrm{q}}=\Delta \dot{\mathcal{X}} \mathrm{q}+\Delta \mathcal{D} \dot{\mathrm{q}}\right)$ indicates clcarly that, in the diagonal gain format of Section I, the diagonal values of $R$ are the inverse of the spring and damping constant values. Hence, a heavy penalty on control, $R$, results in a small control effect.

\section{Physical Interoretation of General Solution}

Theorem 2 provides significant insight into the physical interpretation of possible optimal control solutions for structural systems. It gives key to the closed-loop (redesigned system) dynamics relationships betwecn what is being minimized and the linal value of the objective function in terms of mass, stiffness and damping of the final system. Of coursc, the final control law (i.e., the structure design change) still depends on the choice of $R, \mathcal{B}, a$, and $b$. By imposing additional constraints to the equation set, other recognizable forms of $\hat{Q}$ and $K$ can be obtained. Examples are as follows:

Example 1: Imposed constraint-- $\hat{\theta}=a \hat{\mathcal{D}}+\mathrm{b} \hat{\mathcal{X}}$. Solution:

$$
\begin{aligned}
& \hat{\mathbf{Q}}=\left[\begin{array}{cc}
2 \mathrm{a} \hat{\mathcal{X}} & \mathbf{a} \mathcal{G} \\
\mathbf{a} \mathcal{G} & 2 \mathrm{~b} \hat{\mathcal{D}}-2 \mathrm{a} \hat{\mathcal{M}}
\end{array}\right] \\
& \mathbf{K}=\left[\begin{array}{cc}
\mathrm{a} \hat{\mathcal{D}}+\mathrm{b} \hat{\mathcal{X}} & \mathbf{a} \hat{\mathcal{M}} \\
\mathrm{a} \hat{\mathcal{M}} & \mathrm{b} \hat{\mathcal{M}}
\end{array}\right]
\end{aligned}
$$

This solution is equivalent to the that of Kojitani and Ikeda presented in open-loop form in (24) and (25).

Example 2: Imposed constraints-- $\hat{\Theta}=b \hat{K}$, and $a=0$. Solution:

$$
\hat{\mathrm{Q}}=\left[\begin{array}{cc}
0 & 0 \\
0 & 2 \mathrm{~b} \hat{\mathcal{D}}
\end{array}\right] \mathbf{K}=\mathbf{b}\left[\begin{array}{cc}
\hat{\mathcal{X}} & 0 \\
0 & \hat{\mathcal{M}}
\end{array}\right]
$$

In this case, the integral of a constant times the Rayleigh Dissipation Function is minimized and the objective function cost is $b$ times the total initial energy.

Example 3: Imposed constraints-- $\hat{\Theta}=b \hat{K}$

$$
\begin{array}{r}
\hat{\mathrm{Q}}=2 \mathrm{a}\left[\begin{array}{cc}
\hat{\mathcal{K}} & \frac{1}{2}(\hat{\mathcal{D}}+\mathcal{G}) \\
\frac{1}{2}(\hat{\mathcal{D}}+\mathcal{G})^{*} & \frac{\mathrm{b}}{-} \hat{\mathcal{D}}-\hat{\mathcal{M}}
\end{array}\right] \\
\mathrm{K}=\mathrm{b}\left[\begin{array}{cc}
\hat{\mathcal{K}} & \frac{\mathrm{a}}{\mathrm{b}} \hat{\mathcal{M}} \\
\frac{\mathrm{a}}{\hat{M}} & \hat{\mathcal{M}}
\end{array}\right]
\end{array}
$$

For positive definite $\mathrm{K}$, (38), the requirements are

$$
\mathrm{b}>0 \text { and }\left|\frac{\mathrm{a}}{\mathrm{b}}\right| \mathrm{I}<\mathcal{M}^{-1} \hat{\mathcal{X}}
$$

Orthogonal modal vectors, $\hat{\Phi}$, may be chosen such that the mass and stiffness matrices are normalized to yield $\hat{\Phi}^{*} \mathcal{M} \hat{\Phi}=I$ and $\hat{\Phi} \hat{\mathcal{X}} \hat{\Phi}=(\hat{\Omega})^{2}$ where $\hat{\Omega}$ is a diagonal matrix with diagonal elements $\hat{\omega}_{1}$, the undamped natural frequencies of the closed-loop system. Substituting these orthogonality properties into (39) yields the condition for positive definite $\mathrm{K}$ to be

$$
\mathrm{b}>0 \text { and } \hat{x}>\left(\frac{\mathrm{a}}{\mathrm{b}}\right)^{2} \mathscr{M} \Rightarrow\left|\frac{\mathrm{a}}{\mathrm{b}}\right|<\hat{\omega}^{2}
$$

where $\hat{\omega}_{0}$ is the smallest eigenvalue of the undamped system.

Consider the single-degree-of-freedom subset of this solution. In order for $\mathrm{K}$ to be positive definite, 


$$
\frac{|\mathrm{a}|}{\mathrm{b}}<\hat{\omega}_{0}=\left|\sqrt{\frac{\hat{k}}{\hat{m}}}\right| \Rightarrow\left|\mathbf{K}_{12}\right|<|\sqrt{\hat{k} \hat{m}}|
$$

where the lower case symbols represent the single degreeof-freedom version of their upper case counterparts. The value of $\mathrm{K}_{12}$ at which this occurs is independent of both a and $b . K_{12}$ is constrained to be less than twice critical damping and (lal/b) is constrained to be smaller than the natural frequency. For $a=0$, the cost is the initial energy in the open-loop system. (The closed-loop system is damped even though damping does not appear in the $\mathrm{K}$ matrix.)

Example 4: Imposed constraints-- $\hat{\theta}=b \hat{K}$ and $\hat{\mathcal{D}}=(2 \mathrm{a} / \mathrm{b}) \hat{\mathcal{M}}$. Solution:

$\hat{\mathrm{Q}}=2 \mathrm{a}\left[\begin{array}{cc}\hat{\mathcal{X}} & \frac{1}{2}(\hat{\mathcal{D}}+\mathcal{G}) \\ \frac{1}{2}\left(\hat{\mathcal{D}}+\mathcal{G}^{*}\right) & \hat{\mathcal{M}}\end{array}\right] \mathrm{K}=\mathrm{b}\left[\begin{array}{cc}\hat{\mathcal{X}} & \frac{\hat{\mathcal{D}}}{2} \\ \frac{\hat{\mathcal{D}}}{2} & \hat{\mathcal{M}}\end{array}\right]$

In this case, if there are no gyroscopic terms, $\hat{K}=(b / 2 a) \hat{Q}$ and from equation (8),

$\mathrm{V}=\frac{1}{2} \mathrm{z} *(0) \mathrm{Kz}(0)=\frac{\mathrm{b}}{4 \mathrm{a}} \mathrm{z} *(0) \hat{\mathrm{Q}} \mathrm{z}(0)=\frac{1}{2} \int_{0}^{-} \mathrm{z} * \hat{\mathrm{Q}} \mathrm{zdt}$

This simple scalar relationship, which requires $a>0$, is interesting in that it is equivalent to the integrand remaining constant for a period of time equal to $b / 2 a$ and then dropping to zero, or dropping linearly to zero over time period b/a.

Example 5: Imposed constraints-- $\hat{\Theta}=\mathbf{a} \hat{\mathcal{D}}+\mathbf{b} \hat{\mathcal{X}}$, and $\hat{\mathcal{D}}=\left(\frac{2 \mathrm{a}}{\mathrm{b}}\right) \mathcal{M}$. Solution:

$\hat{\mathrm{Q}}=2 \mathrm{a}\left[\begin{array}{cc}\hat{\mathcal{X}} & 0 \\ 0 & \hat{\mathcal{M}}\end{array}\right] \mathrm{K}=\mathrm{b}\left[\begin{array}{cc}\hat{\mathcal{K}}+2\left(\frac{\mathrm{a}}{\mathrm{b}}\right)^{2} \hat{\mathcal{M}} & \frac{\mathrm{a}}{\mathbf{b}} \\ \frac{\mathrm{a}}{\hat{M}} & \hat{\mathcal{M}}\end{array}\right]$

In this case, the integral of the total energy is minimized.

Example 6: Imposed constraints-- $\hat{\theta}$ arbitrary and $a=0$

$\hat{\mathrm{Q}}=\left[\begin{array}{cc}0 & (-\hat{\Theta}+\mathrm{b} \hat{\mathcal{X}}) \\ (-\hat{\Theta}+\mathrm{b} \hat{\mathcal{X}}) & 2 \mathrm{~b} \hat{\mathcal{D}}\end{array}\right] \mathrm{K}=\mathrm{b}\left[\begin{array}{cc}\hat{\Theta} & 0 \\ 0 & \mathrm{~b} \hat{\mathcal{M}}\end{array}\right]$

In this case, note that if $b=1$ and $\hat{\Theta}=I$, the final cost, which is minimal for any particular $B$ and $R$, is the sum of the kinetic energy and the squared displacements. This has the potential of being a highly useful physical case where practical solutions exist.
Example 7: The solution of Belvin and Park, (29) through (32), is not a member of this solution set in general because it assumes values for all four terms of $\mathrm{Q}_{\mathrm{ij}}$ and for $\Gamma$. The resulting $K_{22}$ is not necessarily constrained to the same predefined connection form although $\mathrm{K}_{12}$ is. Equivalence occurs in the following special case:

$$
\mathcal{K}=\frac{(2 a+b)}{b^{2}} \mathcal{M}, \eta=a, \hat{\Theta}=2 b \mathcal{X}
$$

Note that the original statement of the Belvin-Park solution required $\mathcal{B}^{-1}$ to exist and $\Gamma=\mathcal{X}$.

\section{$V$ Eptimal Values of the $R$ Weighting Matrix/Control_Gain}

In the general LQR control design problem, choosing the control weighting matrix $R$ (or $\Gamma$ if the connection matrix $\mathcal{B}$ is not predefined) is a difficult task. In the closed-form solution of Theorem 3, a permissible

set of $Q_{i j}(Q \geq 0)$ and/or $\hat{\Theta}$ matrices can be selected based on the physics of the problem, and the equations solved for $\Gamma$, the optimal feedback gain to minimize the resulting function. Solutions, where they exist, indicate what the ideal control would be but are not always physically practical. The following subsections illustrate this process.

\section{Optimal R for Specified Open-Loop O}

Rewriting the $Q$ equations of (37) gives insight into selection options for final solutions in terms of frequently used weighting matrix forms, where they exist.

$$
\begin{gathered}
\mathrm{Q}_{11}=2 \mathrm{a} \mathcal{X}+\mathrm{a}^{2} \Gamma \\
\mathrm{Q}_{12}=-\hat{\Theta}+\mathrm{b} \mathcal{X}+\mathrm{a}(\mathcal{D}+\mathcal{G})+\mathrm{ab} \Gamma \\
\mathrm{Q}_{22}=2 \mathrm{~b} \mathcal{D}+\mathrm{b}^{2} \Gamma-2 \mathrm{a} \hat{\mathcal{M}}
\end{gathered}
$$

Having already chosen two of the four parameter matrices allowable for a unique solution, only two remain. From (51) to (53) it is clear that one choice is to choose either $Q_{11}$ or $Q_{22}$, which fixes $\Gamma$, and either $Q_{12}$ or $\hat{\Theta}$ (i.e., $K_{11}$ ), either of which fixes the other. (Note that $Q_{12}$ must contain a term which equals the gyroscopic term $\mathrm{a} \mathcal{G}$, if it exists, since all other matrices are symmetric and $\Gamma$ is defined to be symmetric.) If the available connectivity, $B$, is known, then the optimal member gain is $R^{-1}$ and is solvable from $\Gamma$. A second possibility it to choose both $Q_{12}$ and $\hat{\Theta}$ which fixes $\Gamma$ and, hence, $Q_{11}$ and $Q_{22}$. The difficulty in doing this is that the closed-loop objective function is of the generalized energy form and, consequently, many frequently used non-energy $Q$ forms produce impractical solutions. 
Choosing both $Q_{11}$ and $Q_{22}$ assumes that an additional constraint equation, obtained by eliminating $\Gamma$ between (51) and (53), is satisfied. This constraint,

$$
Q_{11}=\frac{a^{2}}{b^{2}}\left(Q_{22}-2 b \mathcal{D}+2 a M\right)+2 a x
$$

implies a fixed relationship between the mass, stiffness, and damping matrices which is not likely to exist initially and may not be readily imposed on the system.

Some examples, based on various choices of parameter matrices are presented below:

Example 8: $Q_{11}=1, Q_{12}=0$

$$
\begin{gathered}
\Gamma=\frac{1}{a^{2}}(I-2 a \mathcal{X}) \\
Q_{22}=2 b \mathcal{D}-2 a \mathcal{M}+\frac{b^{2}}{a^{2}}(I-2 a X) \\
\hat{\Theta}=-b \mathcal{X}+a \mathcal{D}+\frac{b}{a} I
\end{gathered}
$$

This solution is easily shown to be physically impractical by examining the closed-loop stiffness matrix.

$$
X+\Delta X=\frac{1}{a} I-X
$$

For most problems, this matrix will require members with negative stiffness. For example, for the three-mass system, Configuration 1 , in Fig. 3, referring to the notation of Fig. 2 and assuming :

$$
G_{k}=[2,2,4,1,3,1] \text { and } a=0.2,
$$

the closed-loop spring gains become

$$
G_{k}=[3,3,1,-1,-3,-1]
$$

with negative spring constants on the ungrounded springs. For the choice of $\mathrm{Q}_{11}=\mathrm{I}, \mathrm{R}$ cannot be positive definite unless the closed=loop stiffness matrix is diagonal, that is, no masses are interconnected.

Example 9: $Q_{12}=a G, \hat{\Theta}=b X$

$$
\begin{gathered}
\Gamma=-\frac{1}{b} \mathcal{D} \\
Q_{11}=-\frac{a^{2}}{b} \mathcal{D}+2 a X \\
Q_{n}=b \mathcal{D}-2 a M
\end{gathered}
$$

In this casc, $\Gamma$, (i.c., $R$ ) is negative and the stiffness and damping changes are

$$
\Delta \mathcal{X}=-\frac{\mathrm{a}}{\mathrm{b}} \mathcal{D}, \text { and } \Delta \mathcal{D}=-\mathcal{D}
$$

The closed-loop damping is zero and the system is not asymptotically stable.

Example 10: $z^{*} Q z=y^{*} y=z^{*} C^{*} C z$

$$
\left(C^{*}=\left[\begin{array}{ll}
C_{1} & C_{2}
\end{array}\right]^{*}\right) \text {, arbitrary } \hat{\theta} \text {. }
$$

This choice fixes all three $Q$ matrices and, hence, imposes a requirement that constraint (54) be satisfied, that is

$C_{1} * C_{1}+\frac{a^{2}}{b^{2}} C_{2} * C_{2}=\frac{a^{2}}{b^{2}}(-2 b \mathcal{D}+2 a \mathcal{M})+2 a X$

Furthermore, (52) becomes

$$
\Gamma=\frac{1}{a b}\left[C_{1} * C_{2}+\hat{\Theta}-b \mathcal{X}-a(\mathcal{D}+G)\right]
$$

which requires $\mathrm{C}_{1} * \mathrm{C}_{2}$ to contain a skew symmetric term to offset the gyroscopic term. Even if the gyroscopic term is zero, the constraint between $C_{1}$ and $C_{2}$ will be difficult if not impossible to meet in order to get a valid solution.

\section{Ontimal R for Specified Closed-Loop Q}

Prespecifying closed-loop parameters in order to solve for the optimal $R$ is an alternative to the open-loop case above. From (35), the appropriate relationships are

$$
\begin{aligned}
\hat{\mathrm{Q}}_{11} & =2 \mathrm{a} \hat{\mathcal{X}}=2 \mathrm{a} \mathcal{X}+2 \mathrm{a}^{2} \Gamma \\
\hat{\mathrm{Q}}_{12} & =-\hat{\Theta}+\mathrm{a}(\hat{\mathcal{D}}+G)+\mathrm{b} \hat{\mathcal{X}} \\
& =-\hat{\Theta}+\mathrm{a}(\mathcal{D}+G)+\mathrm{b} \mathcal{X}+2 \mathrm{ab} \Gamma \\
\hat{\mathrm{Q}}_{2} & =2 \mathrm{~b} \hat{\mathcal{D}}-2 \mathrm{a} \hat{\mathcal{M}}=2 \mathrm{~b} \mathcal{D}-2 \mathrm{a} \hat{\mathcal{M}}+2 \mathrm{~b}^{2} \Gamma
\end{aligned}
$$

Example 11: $\hat{\mathrm{Q}}_{11}=I$, Arbitrary second choice.

In this case, we are minimizing the square of the closed-loop displacements. The closed-loop system requires

$$
\hat{x}=\frac{1}{2 a} I
$$

That is, the closed-loop stiffness is diagonal, no matter what its open-loop form. Clearly, from the diagonal gain conceptualization of Section I, the solution is decentralized control with all masses independently grounded (and all interconnections eliminated). However, this is rarely practical in passive systems, if ever. In active systems, considerable energy must be expended to cancel the internal forces of the connecting springs and dampers, an inefficient approach at best. Of course, removing the 
interconnections and applying independent, decentralized, active controls (i.e. "flying in formation") will work but is also likely to be impractical.

\section{Concluding Remarks}

The design of dissipative structure/control systems using linear quadratic regulator (LQR) theory is investigated. Results are summarized as follows:

1) The problem is formulated in physical coordinates, in term of mass, stiffness, and damping matrices. A diagonal-gain structural connectivity form is used to clarify physical interpretation.

2) Solutions to the algebraic Riccati equation are developed which constrain system changes to predefined, physically possible connections in mechanical systems.

3) A general closed-form solution is presented which can be adapted to many $L Q R$ objective functions. The closed-loop objective function is a particular generalized energy form, regardless of what open-loop weighting matrix $(Q)$ form is chosen.

4) An optimal control gain weighting matrix (R) is shown to be available for properly specified objective functions. Improper choices of Q weighting matrices are shown to result in impractical hidden constraints or physically non-existent solutions for control gains.

\section{Beferences}

1. Hanks, B. R., and Skelton, R. E.: An Approach for Designing Structures by Modern Control Theory. Presented at the 24th AIAA/ASME/AHS Structures, Structural Dynamics, and Materials Conference, Lake Tahoc, Nevada, May 1983. AIAA Paper 83-0815.

2. Hvorat, D.: A Class of Active LQG Optimal Actuators. Automatica, Vol. 18, No. 1, pp. 117-119, 1982.

3. Skclton, R.E., and Hanks, B. R.: Structure Redesign for Improved Dynamic Response, Presented at the 1990 American Control Conference, San Diego, California, May, 1990. NASA-TM-102673

4. Skelton, R. E., Hanks, B. R. and Smith, M.: Structure Redesign for Improved Dynamic Response, to appcar.

5. Skelton, R. E.: Dynamic System Control, Linear Systems Analysis and Synthesis. John Wiley \& Sons, 1988.

6. Kojitani, K. and Ikeda, M.: Optimal Control of Large Space Structures Using Collocated Feedback. Private communication to R. E. Skelton, Fcbruary, 1988.
7. Belvin, W. K.; and Park, K. C.: Structural Tailoring and Fcedback Control Synthesis: An Interdisciplinary Approach. AIAA Journal of Guidance, Control, and Dynamics, Vol. 13, No. 3, pp. 424-May-June, 1990.

8. Inman, D. J.: Critical Damping in Complex Structures and Control Systems. Vibration Damping Workshop 1984 Proccedings, AFWAL TR-84-3064, pp AAA-1-11, November 1984 . 


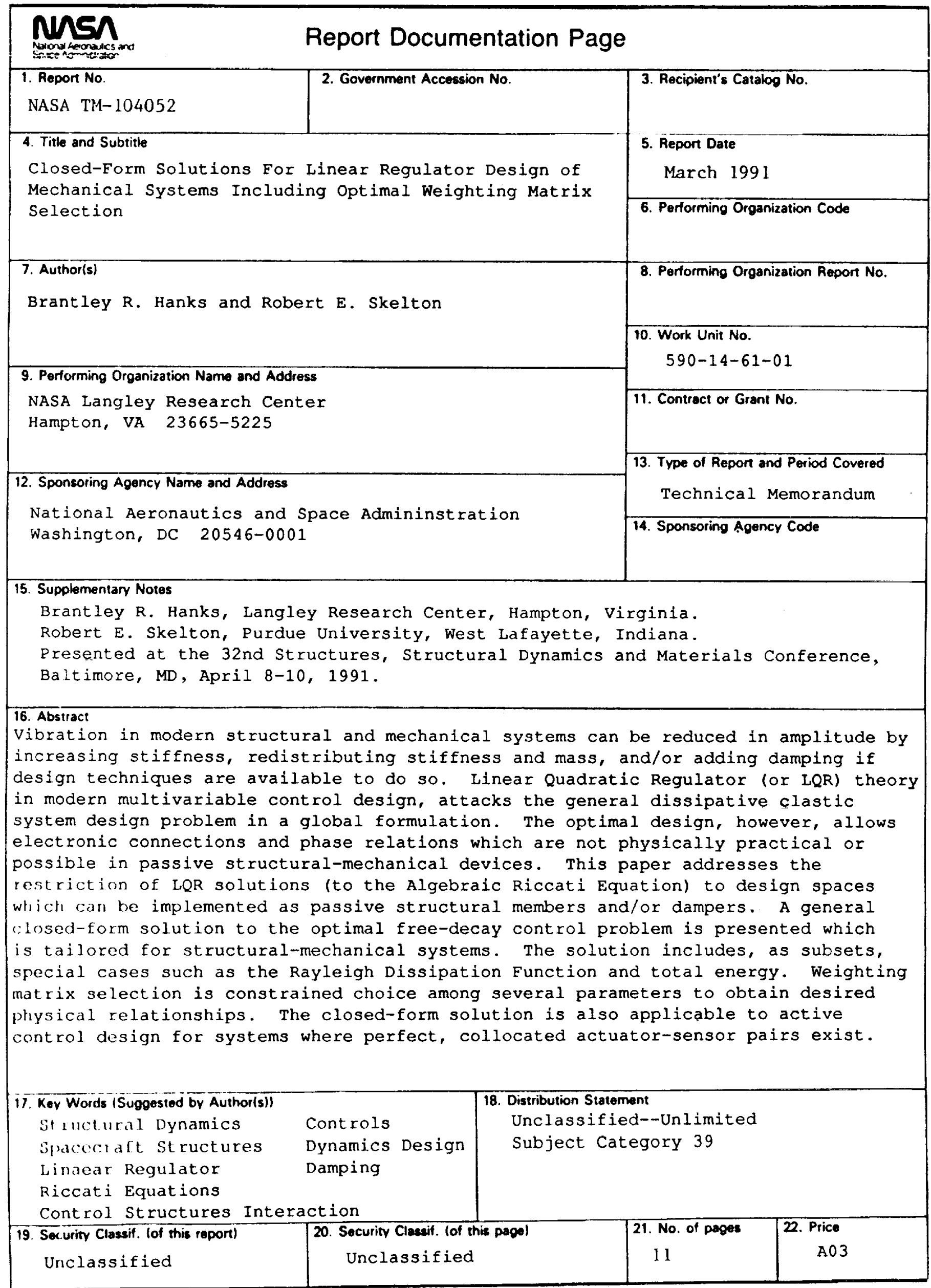


